\title{
What do day hospital attenders really want?
}

\section{A survey of patient preferences}

\author{
Denise Riordan and Louis Appleby
}

\begin{abstract}
The views of psychiatric day hospltal patients on the helpfulness of components of their treatment programme were surveyed. Ratings given to social activities were significantty higher than those given to therapeutic activities. Patients rated having a bus pass and access to hairdressing services as the most helpful parts of day hosplital attendance while community meetings and ward round attendance were regarded as the least helpful. Ratings were, however, generally favourable. No significant effect of patient age, gender, or duration of attendance was found. Possible explanations for these preferences are discussed. Patient preferences such as these are likely to influence purchasers of care, and thus the use of resources by providers.
\end{abstract}

The psychiatric day hospital is set to assume increasing importance in the provision of mental health care. Following NHS reforms, both purchasers and providers are likely to seek forms of care other than the in-patient ward, and the day hospital can expect to provide for the acutely ill (Creed et al, 1989), as well as its more traditional patient group who suffer from chronic disorders. In doing so it will have to meet the needs of patients of all ages.

As a consumer-led health service develops, patient preferences are of particular importance, and the attitudes and preferences of patients are in themselves an area worthy of study. McIntyre et al (1989) showed that the therapeutic experiences that in-patients valued most were talking to caregivers such as the doctor or nurse, whereas social experiences such as mixing with people were not considered to be valuable. In contrast, studies of day hospital patients have suggested that 'non-specific' components of attendance such as getting out of the house and mixing with people are highly rated (Baker et al, 1986; Hsu et al, 1983). These studies have looked only at newly referred attenders of the day hospital and not at longterm attenders.

The aim of this study was to identify those aspects of day hospital care that patients consider most helpful. The study also tests the hypothesis that long-term (i.e. chronic) and older patients will rate 'social factors' in a day hospital programme (i.e. supportive, practical and social activities with no direct therapeutic purpose) more highly than 'therapeutic factors' (i.e. directed at acute or sub-acute clinical problems).

\section{The study}

The subjects of the study were patients attending the psychiatric day hospital at Withington Hospital, a large teaching hospital with a catchment area psychiatric service. All patients on current patient lists were contacted by letter and asked to take part in a ten minute interview. Interviews were carried over a one-week period in June 1993. Age, gender, diagnosis and duration of attendance were recorded from the casenotes. At interview patients were asked to list a number of components of their treatment programme on a 5-point scale where $0=$ not at all helpful and $4=$ extremely helpful.

The items were divided into therapeutic and social factors as shown, and in the interview items were worded as listed below.

\section{Therapeutic factor actors}

Talking to a doctor; talking to a nurse; receiving medication; going to the ward round: having an opportunity to discuss problems; having family interviews (i.e. where relevant family members meet with the staff 
Table 1. Therapeutic factors

\begin{tabular}{|c|c|c|c|c|c|c|c|}
\hline \multirow{2}{*}{ Therapeutic foctors } & \multirow{2}{*}{$\begin{array}{l}n \text { (number of } \\
\text { respondents) }\end{array}$} & \multicolumn{5}{|c|}{ Proportion giving score (as percentage of $n$ ) } & \multirow[t]{2}{*}{ Mean } \\
\hline & & $\mathbf{0}$ & 1 & 2 & 3 & 4 & \\
\hline Discussing problems & 81 & 0 & 7 & 11 & 40 & 42 & 3.16 \\
\hline Obtaining sick note & 23 & 4 & 9 & 4 & 39 & 44 & 3.09 \\
\hline Home visits & 29 & 7 & 3 & 17 & 35 & 38 & 2.93 \\
\hline Talking to nurse & 82 & 2 & 12 & 16 & $\mathbf{3 1}$ & 39 & 2.91 \\
\hline Occupational therapy & 41 & 5 & 5 & 22 & 44 & 24 & 2.78 \\
\hline Group therapy & 57 & 2 & 11 & 26 & 40 & 21 & 2.68 \\
\hline Talking to doctor & 68 & 7 & 15 & 25 & 28 & 25 & 2.48 \\
\hline Recelving medication & 63 & 8 & 14 & 27 & 25 & 25 & 2.43 \\
\hline Relaxation therapy & 25 & 17 & 8 & 20 & 29 & 26 & 2.37 \\
\hline Family meetings & 19 & 16 & 16 & 20 & 16 & 32 & 2.31 \\
\hline Ward round & 57 & 14 & 25 & 19 & 26 & 16 & 2.05 \\
\hline Community meeting & 46 & 15 & 24 & 28 & 26 & 7 & 1.85 \\
\hline
\end{tabular}

Median scores are in bold type

and patient to discuss issues related to the patients illness); home visits by staff; relaxation therapy; group therapy; community meetings (at which patients and staff discuss issues related to the running of the day hospital); occupational therapy; and having sick notes issued.

\section{Social factors}

Getting out of the house; mixing with other people; taking part in social activities; going on outings; recetving meals; having a bus pass; and having access to hospital hairdressing services.

\section{Statistical analysis}

The results were analysed using unpaired $t$-tests and a one-way analysis of variance.

\section{Findings}

Of 114 day hospital attenders, 84 (74\%) took part in the study; $40(48 \%)$ were male and 44 (52\%) female. The mean age was 48.4 years (range 21-85) and the mean period of attendance 34.6 months (range 1-180 months).

\section{Most and least popular activities}

Tables 1 and 2 show the mean ratings for each activity on the 0-4 scale described above. Median scores are in bold type. The figures in the first column represent the number of respondents who reported taking part in each activity.

The mean score for social factors was 3.05, while the mean score for therapeutic factors was 2.59; the difference was statistically significant $(P=0.03)$. Factors percetved as most helpful were having a bus pass and access to hairdressing services. Factors rated as least helpful were community meetings and

Table 2. Social factors

\begin{tabular}{|c|c|c|c|c|c|c|c|}
\hline \multirow{2}{*}{ Social factors } & \multirow{2}{*}{$\begin{array}{l}\text { n (number of } \\
\text { respondents) }\end{array}$} & \multicolumn{5}{|c|}{ Proportion giving score (as percentage of $n$ ) } & \multirow[t]{2}{*}{ Mean } \\
\hline & & $\mathbf{0}$ & 1 & 2 & 3 & 4 & \\
\hline & 60 & 0 & 3 & 5 & 18 & 73 & 3.62 \\
\hline $\begin{array}{l}\text { Hoving a bus pass } \\
\text { Going to the hardresser } \\
\text { Getting out of the house } \\
\text { Going on outings } \\
\text { Mixing with others } \\
\text { Social activities } \\
\text { Receiving meals }\end{array}$ & $\begin{array}{l}52 \\
80 \\
61 \\
80 \\
57 \\
68\end{array}$ & $\begin{array}{l}0 \\
0 \\
0 \\
5 \\
4 \\
9\end{array}$ & $\begin{array}{r}2 \\
9 \\
3 \\
9 \\
18 \\
9\end{array}$ & $\begin{array}{r}10 \\
8 \\
18 \\
21 \\
19 \\
19\end{array}$ & $\begin{array}{l}35 \\
\mathbf{3 9} \\
\mathbf{3 8} \\
\mathbf{3 5} \\
\mathbf{3 6} \\
\mathbf{4 1}\end{array}$ & $\begin{array}{l}54 \\
45 \\
41 \\
30 \\
25 \\
22\end{array}$ & $\begin{array}{l}3.40 \\
3.20 \\
3.16 \\
2.76 \\
2.60 \\
2.59\end{array}$ \\
\hline
\end{tabular}

Median scores are in bold type 
the ward round. Talking to a nurse and talking to a doctor received intermediate mean scores, talking to a nurse being rated as more helpful (difference not significant).

\section{Chronicity of attendance}

Duration of attendance was divided into short term (less than 12 months, 33 people), medium term (13-36 months; 17 people) and long term (more than 3 years; 34 people). There was no relationship between chronicity of attendance and valuing any specific social or therapeutic factor, or valuing one group of factors.

\section{Gender}

Social factors were rated more highly by females than males (mean scores 3.09 and 2.97 respectively), but the difference was not significant. Females also rated therapeutic factors more highly than males (mean scores 2.70 and 2.55 respectively) but again this difference was not significant.

\section{Age}

Patients were divided into three age groups: young (21-34 years), middle-aged (35-65 years) and elderly (over 65 years). All age groups rated social factors more highly than therapeutic factors. In both groups of factors, middle-aged attenders gave higher ratings. However, a one-way analysis of variance showed the effect of age to be nonsignificant.

\section{Comment}

The principal finding in this study is that day hospital attenders value social aspects of their care significantly more highly than experiences that are more directly therapeutic. This is in contrast to a study of psychiatric inpatients, who valued therapeutic factors more highly (McIntyre et al, 1989). The study also shows a generally high level of satisfaction with the services provided by the day hospital. $A$ rating of 3 is defined in our study as very helpful' while 2 is defined as 'quite helpful'. The mean score for social factors was 3.05 , and for therapeutic factors 2.59. This high regard for all aspects of day hospital care is in keeping with earlier findings (Baker et al, 1986; Hsu et al, 1983).

The study does not confirm our hypothesis that long-term and older attenders at the day hospital would be more likely to rate social factors more highly than therapeutic ones. It does not show any significant effects of age or sex of attenders. The higher ratings given to social factors were found in all age groups, both sexes and in short-, medium- and longterm attenders.

Some points about the methods used in this study should be made. First, the value of a particular clinical factor, as perceived by the patient, does not necessarily reflect the actual value of that activity. Some experiences, such as attending the ward round, may determine access to others but be regarded as of limited value in themselves. Second, people who do not like what the day hospital can offer are unlikely to attend; the study population is therefore self selected for positive attitudes to the location of care. Third, those interviewed may have felt obliged to rate parts of their programme as positive. Fourth, no assessment of the test-retest reliability of the scale used in the study was made. Fifth, the apparent absence of an effect of age or gender may reflect insensitivity of the scale.

Nevertheless, the scale has obvious face validity and the response rate was high. Despite any biases in the subject sample, consistent differences were found between the groups of factors and these were statistically significant in the overall study population. Two questions therefore arise from the results; how can these differences be explained? is the patients' preference that they reflect an acceptable basis for day hospital attendance?

Several explanations are possible for the greater perceived usefulness of social factors. Therapeutic factors may be seen as confirming illness and may therefore be less valued than social activities that appear to confirm normality. This may explain the contrast to inpatients in the study by McIntyre et al (1989) who, having accepted illness status by residing in hospital, may then have seen therapeutic factors as a route to recovery. Therapeutic activities such as group therapy may also confront patients with difficulties they must learn to overcome. The fact that discussing problems' is the most valued therapeutic factor supports the idea that the least challenging therapies are the most acceptable to patients though of course they are not necessarily the most effective. In addition, patients who are facing stressors at home may particularly value the element of asylum in day hospital attendance represented in some social factors.

Given the increasing need to justify the use 
of resources, it could be argued that social activities could be provided in a setting less expensive than a day hospital. This might relieve pressure on day hospital places which could then be used for more acutely ill patients. However, social activities may help patients to accept other parts of their therapeutic programme. In addition, the results of this survey suggests that a loss of social activities from the psychiatric day hospital would not be in keeping with patient preferences-an increasing influence on purchasers of care.

\section{References}

Baker, G., Gardiner, B., Perez-Gil., J., et al (1986) Psychiatric day hospitals: the patients and their preferences in treatment. International Joumal of Social Psychiatry. 32, 64-72.
Creed, F. Godbert, K. \& Huxley, P. (1989) Treatment of severe psychiatric illness in a day hospital. British Joumal of Psychiatry, 154, 341-347.

HSU, L. RidLEY, C. \& Hinde, R. (1983) How do psychiatric patients view their own treatment: a study of $\mathbf{5 0}$ day hospital patients. International Journal of Social Psychiatry, 29, 60-69.

MCINTYRE, K. FARRELL. M. \& DAVID, A. (1989) What do psychiatric inpatients really want? British Medicine Journal, 298, 159-160.

* Denise Riordan, Senior Registrar in Child \& Adolescent Psychiatry, Royal Manchester Children's Hospital, Pendlebury M27 1HA; and Louis Appleby, Senior Lecturer in Psychiatry, Withington Hospital, Manchester M20 8LR:

*Correspondence

\section{Psychiatric Aspects of Physical Disease}

\section{Edited by Allan House, Richard Mayou \& Christopher Mallinson}

This book describes the extent and nature of psychiatric problems associated with physical illness and the ways in which both can be managed by physicians and psychiatrists. Examples are given of simple measures that can be incorporated into routine care and of occasions where specialist referral is appropriate. The book comprises edited papers based on a joint conference of the Royal Colleges of Physicians and Psychiatrists together with additional editorial sections. It provides a sister volume to Medical Symptoms not Explained by Organic Disease and complements the joint report of the two Colleges on Psychological Care of Medical Patients: Recognition of Need and Service Provision.

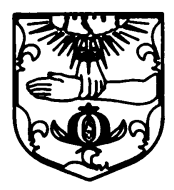

$\bullet £ 15.00 \bullet 110$ pp. $\bullet 1995 \bullet$ ISBN 1873240929

Available from bookshops and from the Publications Department, Royal College of Psychiatrists, 17 Belgrave Square, London SW1X 8PG (Tel. 0171-235 2351, extension 146)

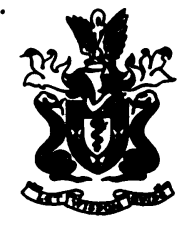

